\title{
Biodegradable Dual Semi-circular Patch Antenna Tile for Smart Floors
}

This paper was downloaded from TechRxiv (https://www.techrxiv.org).

\section{LICENSE}

CC BY 4.0

SUBMISSION DATE / POSTED DATE

$16-11-2020 / 13-01-2021$

\section{CITATION}

Fazeli Khalili, Hossein; Vande Ginste, Dries; Rogier, Hendrik (2021): Biodegradable Dual Semi-circular Patch Antenna Tile for Smart Floors. TechRxiv. Preprint. https://doi.org/10.36227/techrxiv.13241555.v1

DOI 


\title{
Biodegradable Dual Semi-circular Patch Antenna Tile for Smart Floors
}

\author{
Hossein Fazeli Khalili, Sam Lemey, Member, IEEE, Olivier Caytan, Thomas Deckmyn, Sam Agneessens, \\ Member, IEEE, Dries Vande Ginste, Senior Member, IEEE, Hendrik Rogier, Senior Member, IEEE
}

\begin{abstract}
A dual semi-circular microstrip patch antenna implemented on a biodegradable substrate is presented for operation in the [863-873]-MHz and [2.4-2.5]-GHz frequency bands. To cover these frequency bands, two semi-circular patches are compactly integrated onto a biodegradable cork tile, commonly found as support in laminate flooring, serving as a substrate. Thereby, the antenna tile may be seamlessly embedded as a sublayer of the floor structure. A higher-order mode is generated by applying via pins in the antenna topology, to produce a conical radiation pattern with a null at broadside and sectoral coverage in the vertical plane. As such, the concealed-floor antenna covers all azimuth angles of arrival in smart houses. The antenna performance is fully validated, also when the tile is covered by different PVC sheets. Owing to the supplementary design margins, the antenna impedance bandwidth remains covered. Moreover, the radiation patterns are measured in various elevation planes. In stand-alone conditions, a radiation efficiency and a maximum gain of $74.3 \%$ and $5.8 \mathrm{dBi}$ at $2.45 \mathrm{GHz}$ and $48.1 \%$ and 2 dBi at $868 \mathrm{MHz}$ are obtained. Its omni-directional coverage in the horizontal plane, stable performance on the inhomogeneous and biocompatible cork substrate and for various inhomogeneous superstrates and its low-profile integration make the proposed antenna an excellent candidate for smart floors and smart houses.
\end{abstract}

Index Terms-biodegradable cork, dual-band microstrip antenna, antenna tile, smart floors, Internet of Things (IoT)

\section{INTRODUCTION}

W ITHIN the Internet of Things (IoT) vision, designers must overcome challenges such as a massive integration of wireless systems based on low-cost, eco-compatible and durable materials. Smart surfaces and smart floors enable the realization of the IoT for indoor communications, providing connectivity everywhere to everyone and everything [1].

The IoT paradigm requires high-performance systems, which can be realized by increasing the number of wireless transceivers. Yet, conventional WiFi devices are not suitable for integration into smart surfaces, as the complete $\mathrm{WiFi}$ system must be compact and invisible to users [1]. Moreover, it must be implemented in an eco-friendly material that is compatible with the construction process of floors, walls or ceilings. Hence, for smart floors, the electronic devices must be implemented in common biocompatible floor materials and in a manner that fits the floor's production process.

This letter proposes the first low-profile, compact, dual semi-circular patch antenna implemented on a cork substrate. The antenna tile facilitates the integration of large numbers of

H. Fazeli Khalili, S. Lemey, O. Caytan, T. Deckmyn, S. Agneessens, D. Vande Ginste and H. Rogier are with the Electromagnetics Group, Department of Information Technology, Ghent University-imec, 9052 Ghent, Belgium (email: hossein.fazelikhalili@ugent.be). remote radio heads into floors for IoT indoor applications. Its compact size enables direct integration into a floor, modularly constructed by tiles of identical size or as a part of a single floor layer containing one or more antenna tiles.

The cork material, serving as a green, biodegradable substrate [2], typically exhibits inhomogeneous material characteristics, producing highly variable dielectric properties. This also holds for typical floor materials in which the antenna tile is integrated. The effect of varying permittivity and loss tangent in all these substrates and superstrates was fully taken into account during the design process. Even after integration inside a wide range of floor assemblies, this ensures full coverage of both the license-free [2.4-2.5]-GHz WLAN (Wireless Local Area Network) band [3], preferred for shortrange wireless communication at high bitrates, and the [863870]-MHz Short SRDs (Short Range Devices) band [4], suited for information exchange over larger ranges at low data rates and low transmit power. Finally, the antenna radiation pattern was shaped to cover all azimuth angles during deployment.

In current related work, [5] proposes a UHF RFID tag antenna embedded in a concrete floor to track the location of a shipyard transporter. The thick plastic housing of the tag antenna disagrees with the eco-friendly approach adopted in the IoT vision. [6] implements an ultra-wideband substrate-integrated-waveguide cavity-backed slot antenna in eco-friendly cork material. The antenna remains operational even when covered by cork and Plexiglas superstrates. Yet, such materials are not commonly used as a floor's superstrate. Moreover, the antennas in [5] and [6] are not dual-band. [7] presents a $5.8 \mathrm{GHz}$ indoor localization antenna combined with a $13.56 \mathrm{MHz}$ energy harvesting coil. Besides that the design is not suited for communication in two different bands, the copper structures are laminated on top of eco-friendly cork and wood materials without a traditional superstrate, and, as such, not suitable for inconspicuous integration into a floor.

In contrast, our antenna tile may be invisibly and robustly integrated into smart floors. It provides sufficient real-estate to incorporate electronic components, such as reaction force [8] and/or pressure sensors [9] [10], to target applications such as user identification [11] and tracking [12], walking trajectory [13] and gait analysis [14], fall or posture detection [15] [16] and elderly care [17] [18]. All required sensing and transceiver electronics may be deployed on the same antenna tile, which may wirelessly exchange sensor data with other tiles and/or a central processing unit. Moreover, the antenna tile may set up a floor-integrated WiFi access point for communication with wearable devices [19] and/or fixed wireless infrastructure, or 


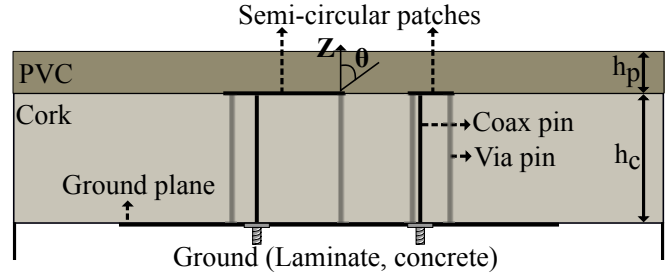

(a)

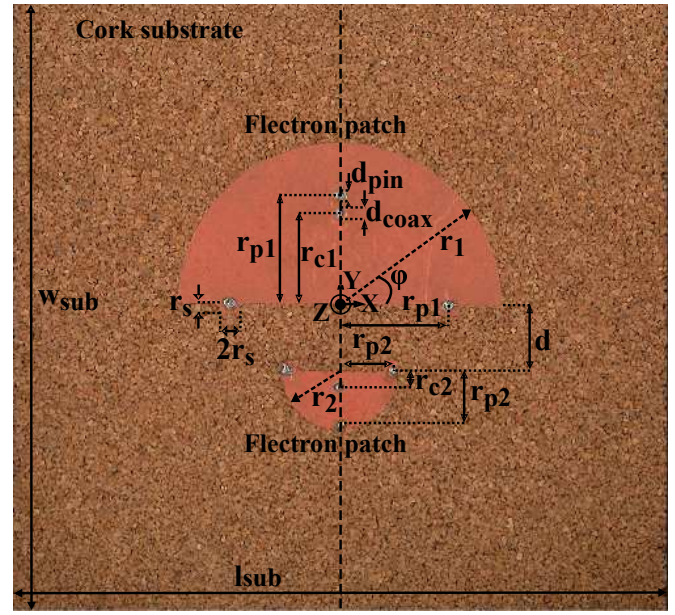

(b)

Figure 1: (a) Antenna tile geometry for integration into smart floors, cork substrate as a floor sub-layer, PVC as a superstrate. $h_{p}=2 \mathrm{~mm}$ and $h_{c}=6 \mathrm{~mm}$. (b) Antenna layer containing two semi-circular patches: $d_{\text {pin }}=2 \mathrm{~mm}, d_{\text {coax }}=1.3 \mathrm{~mm}, r_{p 1}=50$ $\mathrm{mm}, r_{c 1}=42 \mathrm{~mm}, r_{1}=74 \mathrm{~mm}, d=30 \mathrm{~mm}, r_{c 2}=7 \mathrm{~mm}, r_{p 2}=25$ $\mathrm{mm}, r_{2}=27 \mathrm{~mm}, r_{s}=4.5 \mathrm{~mm}$ and $w_{s u b}=l_{s u b}=300 \mathrm{~mm}$.

for indoor positioning via WiFi fingerprinting [20].

Where Section II concentrates on the design requirements for a typical smart floor antenna, the antenna topology and the design process, Section III presents the simulated and measured results. Section IV concludes this letter.

\section{ANTENNA DESIGN AND FABRICATION}

\section{A. Design considerations}

To protect the environment and to reduce waste, materials must be used that do not harm our environment. Cork is such an eco-friendly and recyclable material. Moreover, its low dielectric constant makes it also suitable as antenna substrate and it can be directly embedded into floors, where the cork material acts as support in one of the floor's sub-layers. We propose a low-profile circular microstrip patch antenna as ideal topology for inconspicuous integration into smart floors. As shown in Fig. 1a, the antenna is designed to meet the requirements when covered by a polyvinyl chloride (PVC) layer, which is a common superstrate in a floor structure, used as protection for the lower layer and also as decoration. Fig. 1b shows the antenna geometry optimized in the design process.

The design challenge consists in making antenna performance resilient to the inhomogeneity of both the cork substrate and PVC superstrate in both targeted frequency bands. This requires additional impedance bandwidth margins and accurate characterization of the antenna materials in the frequency bands of interest. For each frequency band, the cork's dielectric constant $\left(\epsilon_{r}\right)$ and loss tangent $(\tan \delta)$ are first estimated by the matrix-pencil two-line method [21]. In the [2.4-2.5]-GHz band, the properties are derived from a 131mm-long and 100-mm-long 26-mm-wide microstrip line, realized in copper-plated Tafetta electroconducting textile (sheet resistance $0.05 \Omega / \mathrm{sq}$ ) on a 6 -mm-thick cork substrate. The measured frequency-averaged values, $\left(\epsilon_{r}, \tan \delta\right)=(1.34,0.03)$, then serve as estimate for a more accurate resonator technique, applied at both operating frequencies. Therefore, three circular patch antennas with four via pins, being the bottom semicircular antenna of Fig. $1 \mathrm{~b}$ and its mirror image around the antenna's top straight edge, are simulated and fabricated. The prototypes' exact substrate permittivity is characterized by matching the measured reflection coefficient $\left|S_{11}\right|$ to the simulated one, yielding for $\left(\epsilon_{r}, \tan \delta\right):(1.235,0.036),(1.255$, $0.030)$ and $(1.210,0.041)$. The average of these values serves as nominal value for the [2.4-2.5]-GHz band: $(1.24,0.035)$.

The procedure is repeated in the lower frequency band, now with 181-mm-long and 100-mm-long 26-mm-wide microstrip lines. As the lower frequency band is quite narrow, the measured average values, being $(1.234,0.019)$, serve as nominal value for the [863-873]-MHz band. When considering both frequency bands simultaneously during the design, the average of both nominal values is applied: $(1.237,0.027)$.

As the PVC material is also inhomogeneous, five 2-mmthick PVC sheets by Flexxfloors [29] are characterized by the matrix-pencil method. Averaged over the lower frequency band, we obtain for $\left(\epsilon_{r}, \tan \delta\right)$ : $(3.43,0.011),(3.76,0.014)$, $(3.61,0.025),(3.85,0.005)$ and $(3.58,0.011)$. During the computer-aided design process, carried out with the CST Microwave Studio frequency-domain simulator, the average of these values is applied: $(3.646,0.013)$.

To account for the inhomogeneity of both substrate and superstrate, we design the antenna for a wider frequency band, making it robust against variations in the cork's permittivity and ensuring stable performance after deployment under the floor's PVC cover sheet. Moreover, the margins also counter fabrication inaccuracies. Specifically, the antenna is optimized for an impedance bandwidth ranging from $2.3 \mathrm{GHz}$ to 2.6$\mathrm{GHz}$, including a margin of at least $200 \mathrm{MHz}$, and from $856 \mathrm{MHz}$ to $880 \mathrm{MHz}$, adding a margin of more than $14 \mathrm{MHz}$. To verify that the supplementary bandwidth margins are sufficient to overcome the inhomogeneity of the cork and PVC, the actual frequency bands must remain covered for the minimum and maximum cork permittivity, measured by the resonator technique, and for the PVC permittivity values. Simulations show that, for a superstrate with an average $\left(\epsilon_{r}\right.$, $\tan \delta)=(3.646,0.013)$, the frequency bands remain covered when the substrate's permittivity varies in the range from 1.19 to 1.31 . For a substrate with average $\left(\epsilon_{r}, \tan \delta\right)=(1.237,0.027)$, the requirements remain satisfied for variations in the PVC superstrate's permittivity from 3.1 to 4.8 .

To guarantee acceptable gain in all azimuth directions and a stable communication link between the floor antenna tile and mobile users, we generate a monopole-like antenna radiation pattern [22], as required for indoor wireless 

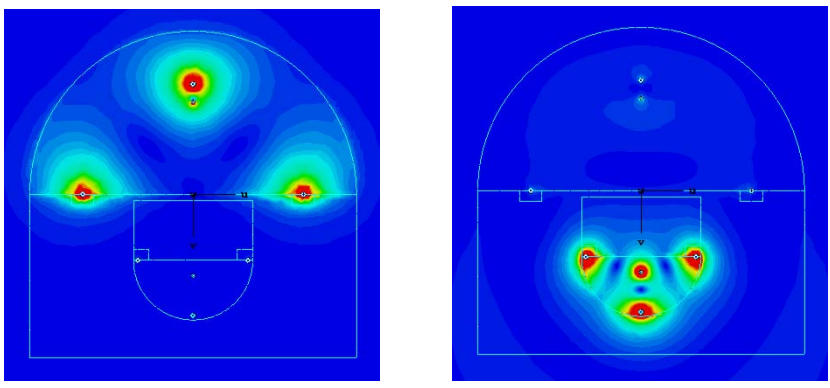

Figure 2: Magnetic field distribution underneath the antenna patches. Left: large patch resonating at $868 \mathrm{MHz}$. Right: small patch resonating at $2.45 \mathrm{GHz}$. The colors are indicative of the field strength.

sensor-to-sensor, infrastructure-to-person and infrastructureto-infrastructure communication. This is obtained in a planar form factor by adopting a novel dual half-mode circular patch antenna topology in which shorting pins are placed, as shown in Fig. 1a. These short-circuit pins modify the current distribution on the patch such that a higher-order mode [23][25] is excited, which generates a radiation pattern with a null along broadside and omni-directional coverage in the horizontal plane for various elevation angles. Fig. 2 shows the magnetic field distributions just underneath the antenna patches at resonance frequencies $868 \mathrm{MHz}$ and $2.45 \mathrm{GHz}$.

Finally, additional sensing and transceiver electronics may be directly integrated onto the antenna tile or embedded in an additional floor layer, which may be textile [13] or polymer based [26]. For the ease of integration and to ensure maximum scalability, the smart tile dimensions are chosen identical to those of common sensor tiles [27] and typical floor tiles.

\section{B. Antenna fabrication process}

As shown in Fig. 1b, two semi-circular patches are implemented on a 6-mm-thick cork substrate, being a composite agglomerate, made of cork granules. Separate antenna patches with individual feeds offer the highest degree of flexibility in terms of interconnections. Both antennas can either be part of a single dual-band wireless system or each individual antenna feed may separately connect to another communication network. Each patch is shorted to the ground plane by three via pins, with an inner and outer radius of $0.8 \mathrm{~mm}$ and $1 \mathrm{~mm}$, resp. The virtual walls in the antenna topology, at the coordinates $y=0, y=-d$ and parallel to XZ-plane, can be approximated as perfect magnetic conductors (PMCs) [28]. These quasiPMC planes enable the miniaturization of the antenna by implementing semi-circular instead of full-circular patches. The via pins are placed at the same distance from each patch center. The ground plane and patches are patterned in Taffeta electro-textile and laminated on the cork substrate through thermally activated glue. The antenna is fed by two coaxial probes with SMA connectors (inner and outer radius $0.65 \mathrm{~mm}$ and $2.05 \mathrm{~mm}$, resp.), soldered to the patches. By adjusting the radius of each patch and the via pin positions, resonance at both $868 \mathrm{MHz}$ and $2.45 \mathrm{GHz}$ is achieved. The feed point

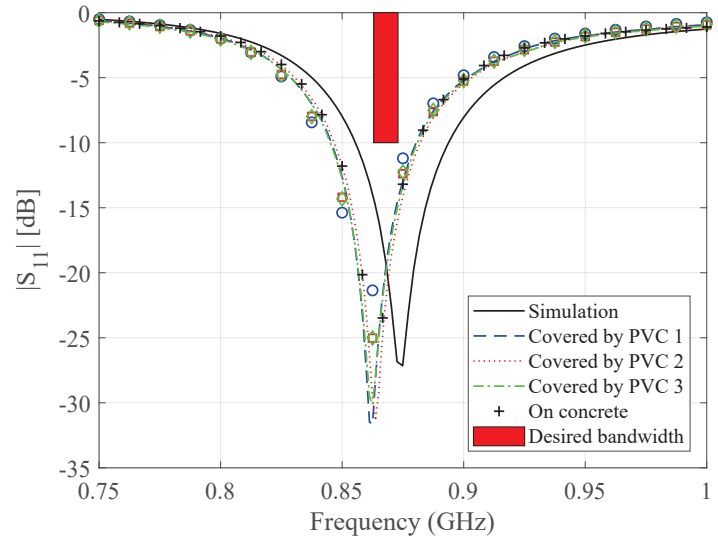

(a)

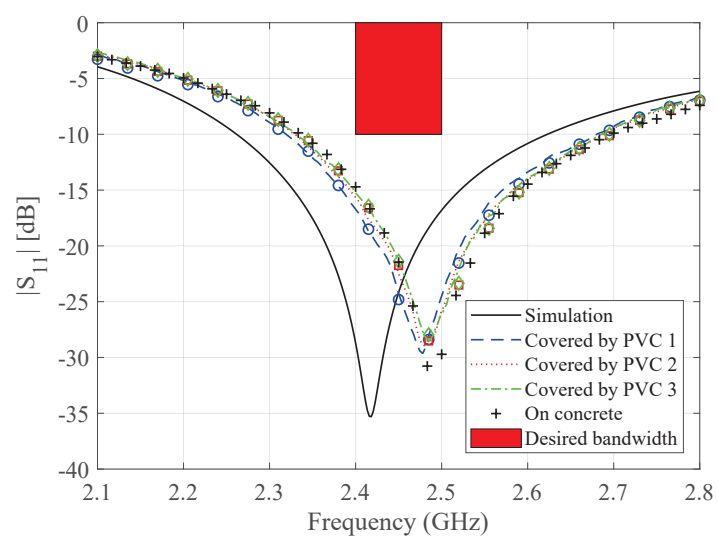

(b)

Figure 3: Input reflection coefficient versus frequency. Simulation in stand-alone condition. Measurement in stand-alone condition (dashed lines), when the antenna is placed on a quasi-PEC plane (markers) and on a concrete support with PVC 2 cover (+), for (a) the larger and (b) the smaller patch.

positions are optimized to obtain impedance matching when the antenna is covered by a 2-mm-thick PVC layer.

\section{RESULTS}

The input reflection coefficients $\left|S_{11}\right|$ of both patches are measured by a Keysight FieldFox N9918A Vector Network Analyzer (VNA), when covering the antenna surface by $30 \mathrm{~cm} \times 30 \mathrm{~cm}$ PVC sheets from three different batches of Flexxfloors Stick Basic Black [29], to verify the antenna specifications in realistic deployment conditions. The simulation and measurement results are shown in Fig. 3. To evaluate the effect of the material backing the antenna tile, three sets of measurements are performed: one set without any backing material, shown as dashed lines in Fig. 3, one with the antenna deployed on concrete tiles and covered by PVC layer 2, shown as black crosses in Fig. 3, and one set with a large perfect electric conductor (PEC) ground plane, serving as a worstcase scenario, plotted with markers in Fig. 3. In the lower frequency band (Fig. 3a), the central resonance frequency has slightly shifted compared to the simulation, due to some small variation in the dielectric constant of the cork substrate and the PVC. However, the antenna's impedance bandwidth of 


\begin{tabular}{|c||c||c||c|}
\hline $\begin{array}{c}\text { Freq. } \\
\text { GHz }\end{array}$ & $\begin{array}{c}\text { Rad. eff. } \\
{[\%]}\end{array}$ & $\begin{array}{c}\text { Beamwidth } \\
\text { degree } \\
\phi=0^{\circ}, 45^{\circ}\end{array}$ & $\begin{array}{c}\text { Max. gain } \\
{[\mathrm{dBi}]} \\
\phi=0^{\circ}, 45^{\circ} \\
\end{array}$ \\
& & $90^{\circ}, 135^{\circ}$ & $90^{\circ}, 135^{\circ}$ \\
\hline 0.863 & $47.2(55.2)$ & $55(54.2), 57(53.3)$ & $0.8(1.0), 0.2(0.9)$ \\
& & $55(53.3), 56(53.5)$ & $1.9(2.2), 0.2(0.9)$ \\
\hline $\mathbf{0 . 8 6 8}$ & $\mathbf{4 8 . 1 ( 5 5 . 7 )}$ & $\mathbf{5 6}(\mathbf{5 4 . 1}), \mathbf{5 6}(\mathbf{5 3 . 2})$ & $\mathbf{0 . 8}(\mathbf{1 . 1}), \mathbf{0 . 4 ( 0 . 9 )}$ \\
& & $\mathbf{5 5}(\mathbf{5 3 . 2}), \mathbf{5 7}(\mathbf{5 3 . 4})$ & $\mathbf{2 . 0}(\mathbf{2 . 3}), \mathbf{0 . 3}(\mathbf{0 . 9})$ \\
\hline 0.873 & $50.1(56.1)$ & $57(55.7), 57(56.2)$ & $0.8(1.2), 0.6(1.0)$ \\
& & $56(53.1), 56(53.4)$ & $2.2(2.4), 0.6(1.0)$ \\
\hline 2.40 & $77.3(81.1)$ & $46(57.4), 31(36.1)$ & $3.4(1.8), 5.1(3.9)$ \\
& & $32(37.0), 29(36.2)$ & $5.4(4.9), 5.3(3.9)$ \\
\hline $\mathbf{2 . 4 5}$ & $\mathbf{7 4 . 3 ( 8 1 . 9 )}$ & $\mathbf{4 3 ( 5 6 . 0 ) , 3 1 ( 3 5 . 9 )}$ & $\mathbf{3 . 2 ( 1 . 8 ) , 4 . 5 ( 3 . 8 )}$ \\
& & $\mathbf{3 2}(\mathbf{3 6 . 2}), \mathbf{2 9}(\mathbf{3 6 . 0})$ & $\mathbf{5 . 8}(\mathbf{5 . 2}), \mathbf{4 . 9}(\mathbf{3 . 8})$ \\
\hline 2.50 & $64.1(82.4)$ & $48(53.0), 31(36.2)$ & $2.6(1.9), 3.5(3.6)$ \\
& & $30(35.6), 28(36.2)$ & $5.7(5.4), 4.0(3.6)$ \\
\hline
\end{tabular}

Table I: Measured and (simulated) radiation performance for the azimuth angles $\phi=0^{\circ}, \phi=45^{\circ}, \phi=90^{\circ}$ and $\phi=135^{\circ}$.

$10 \mathrm{MHz}$ remains perfectly covered. In Fig. 3b, the central resonance frequency has also slightly shifted, due to the same reason. Yet, the antenna still covers the complete $2.45-\mathrm{GHz}$ ISM band. For both bands, the backing material does not affect the antenna's impedance bandwidth, owing to the large antenna ground plane.

The performance of the antenna, covered by a PVC substrate, is further validated by an Agilent N5242A PNA-X VNA and an NSI-MI spherical near-field scanner in an anechoic chamber. To verify all design requirements, the antenna's farfield radiation pattern, radiation efficiency, beamwidth and maximum gain are measured by gain comparison with respect to R\&S HF906 and NSI-RF-SG975 horn reference antennas for the higher and lower frequency bands, resp. The gain patterns at $868 \mathrm{MHz}$ and $2.45 \mathrm{GHz}$ are shown in Fig. 4 for $\phi=0^{\circ}, 45^{\circ}, 90^{\circ}$ and $135^{\circ}$, to ensure that the antenna radiation pattern covers all azimuth angles $\phi$ with sufficient gain in realistic deployment conditions. The measured radiation patterns are almost identical to the simulations, proving that all azimuth directions are perfectly covered for various elevation angles. Moreover, when deploying the antenna tile on a 6-cm-thick concrete supporting layer, Fig. 4 shows that the simulated radiation patterns are not significantly affected, while the antenna gain only slightly decreases due to the presence of the concrete. Next, measurements are carried out at six frequencies in both frequency bands of interest. The radiation efficiency, beam width and maximum gain for each defined elevation plane and frequency are summarized in Table I. The average radiation efficiency equals $71.9 \%$ and $48.4 \%$ in the higher and lower frequency band, resp. The [min, max] antenna gain at $2.45 \mathrm{GHz}$ and $868 \mathrm{MHz}$ amounts to $[3.2,5.8]$ $\mathrm{dBi}$ and $[0.3,0.8] \mathrm{dBi}$, resp.

Despite the miniaturization and the unconventional antenna materials, the antenna still achieves resilient coverage of two frequency bands, as well as robust and high radiation performance. Therefore, it is perfectly applicable for smart floor assemblies in a wide variety of floor types for indoor communications, where the antenna resides near mobile users.

\section{CONCLUSION}

A dual-band microstrip patch antenna for integration in smart floors was designed, realized and validated. This antenna
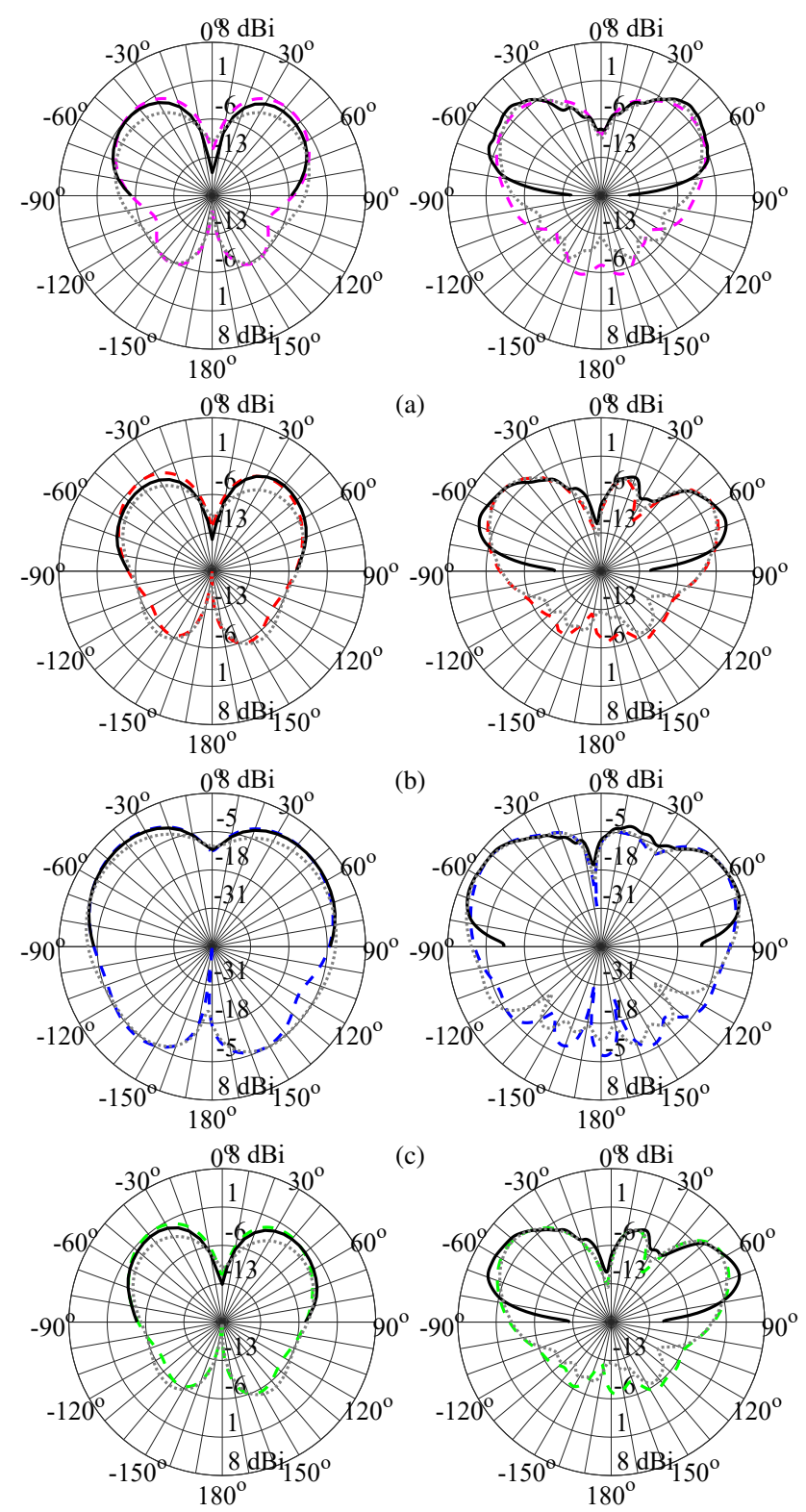

(d)

Figure 4: Measured stand-alone (-), simulated stand-alone ( $-\ldots$ ) and simulated deployed on a 6-cm-thick concrete layer (- - ) antenna gain patterns at $868 \mathrm{MHz}$ (left column) and at $2.45 \mathrm{GHz}$ (right column) for (a) $\phi=0^{\circ}$, (b) $\phi=45^{\circ}$, (c) $\phi=90^{\circ}$ and (d) $\phi=135^{\circ}$.

is implemented in a biocompatible cork material, typically applied in laminate floors. Despite the cork's inhomogeneity, the designed antenna excellently covers the frequency bands of interest, owing to the additional impedance bandwidth margins imposed during its design. Its performance remains insensitive to the inhomogeneity of various PVC superstrates and materials backing the antenna tile. The antenna radiation pattern provides omni-directional coverage in the horizontal plane and sufficient gain at various elevation angles. Its invisible integration into the low-cost and eco-compatible floor material and full coverage of the WLAN [2.4-2.5]-GHz and [863-873]$\mathrm{MHz}$ bands make the antenna tile a perfect candidate for smart surfaces, implementing indoor communication for the IoT. 


\section{REFERENCES}

[1] L. Roselli et al., "Smart Surfaces: Large Area Electronics Systems for Internet of Things Enabled by Energy Harvesting," in Proceedings of the IEEE, vol. 102, no. 11, pp. 1723-1746, Nov. 2014.

[2] D.C. Santos, L. Silva, A. Albuquerque, R. Simões, A.C. Gomes, "Biodegradability enhancement and detoxification of cork processing wastewater molecular size fractions by ozone," Bioresource Technology, vol. 147, pp. 143-151, Nov. 2013.

[3] IEEE 802.11-2007, "Wireless LAN Medium Access Control (MAC) and Physical Layer (PHY) specifications," IEEE, Mar. 2007.

[4] ERC Recommendation 70-03, "Relating to the use of Short Range Devices (SRD)," Electronic Communications Committee, Oct. 2017.

[5] S.H. Jeong and H.W. Son, "UHF RFID Tag Antenna for Embedded Use in a Concrete Floor," IEEE Antennas and Wireless Propagation Letters, vol. 10, pp. 1158-1161, Oct. 2011.

[6] O. Caytan et al., "Half-Mode Substrate-Integrated-Waveguide CavityBacked Slot Antenna on Cork Substrate," IEEE Antennas and Wireless Propagation Letters, vol. 15, pp. 162-165, May. 2016.

[7] C. Mariotti, R. Gonçalves, L. Roselli, N.B. Carvalho and P. Piño, "“Energy evaporation": The new concept of indoor systems for WPT and EH embedded into the floor," 2015 IEEE MTT-S International Microwave Symposium, Phoenix, AZ, 2015, pp. 1-4.

[8] R.J. Orr and G.D. Abowd, "The Smart Floor: A Mechanism for Natural User Identification and Tracking," Proc. 2000 Conf. Human Factors in Computing Systems (CHI 2000), ACM Press, New York, 2000.

[9] W. Liau, C. Wu and L. Fu, "Inhabitants Tracking System in a Cluttered Home Environment Via Floor Load Sensors," IEEE Trans. on Automation Science and Engineering, vol. 5, no. 1, pp. 10-20, Jan. 2008.

[10] M. Andries, O. Simonin and F. Charpillet, "Localization of Humans, Objects, and Robots Interacting on Load-Sensing Floors," IEEE Sensors Journal, vol. 16, no. 4, pp. 1026-1037, Feb. 15, 2016.

[11] R. Vera-Rodriguez, J. S. D. Mason, J. Fierrez and J. Ortega-Garcia, "Comparative Analysis and Fusion of Spatiotemporal Information for Footstep Recognition," IEEE Transactions on Pattern Analysis and Machine Intelligence, vol. 35, no. 4, pp. 823-834, April 2013.

[12] I. Al-Naimi and C. B. Wong, "Indoor human detection and tracking using advanced smart floor," 2017 8th International Conference on Information and Communication Systems (ICICS), Irbid, 2017, pp. 3439.

[13] C. Lauterbach, A. Steinhage and A. Techmer, "Large-area wireless sensor system based on smart textiles," International Multi-Conference on Systems, Signals and Devices, Chemnitz, 2012, pp. 1-2.

[14] D. Bales et al., "Gender Classification of Walkers via Underfloor Accelerometer Measurements," IEEE Internet of Things Journal, vol. 3, no. 6, pp. 1259-1266, Dec. 2016.

[15] M. Daher, A. Diab, M. El Badaoui El Najjar, M. Ali Khalil and F. Charpillet, "Elder Tracking and Fall Detection System Using Smart Tiles," IEEE Sensors Journal, vol. 17, no. 2, pp. 469-479, Jan. 15, 2017.

[16] O. Tanaka, T. Ryu, A. Hayashida, V.G. Moshnyaga, K. Hashimoto (2015) "A Smart Carpet Design for Monitoring People with Dementia". In: Selvaraj H., Zydek D., Chmaj G. (eds) Progress in Systems Engineering. Advances in Intelligent Systems and Computing, vol. 366, Springer, Cham.

[17] N. K. Suryadevara and S. C. Mukhopadhyay, "Wireless Sensor Network Based Home Monitoring System for Wellness Determination of Elderly," IEEE Sensors Journal, vol. 12, no. 6, pp. 1965-1972, June 2012.

[18] A. Gaddam, S. C. Mukhopadhyay and G. S. Gupta, "Elder Care Based on Cognitive Sensor Network," IEEE Sensors Journal, vol. 11, no. 3, pp. 574-581, March 2011.

[19] S. Lemey, S. Agneessens, P. Van Torre, K. Baes, J. Vanfleteren and H. Rogier, "Wearable Flexible Lightweight Modular RFID Tag With Integrated Energy Harvester," IEEE Transactions on Microwave Theory and Techniques, vol. 64, no. 7, pp. 2304-2314, July 2016.

[20] F. Zhao, H. Luo, X. Zhao, Z. Pang and H. Park, "HYFI: Hybrid Floor Identification Based on Wireless Fingerprinting and Barometric Pressure," IEEE Transactions on Industrial Informatics, vol. 13, no. 1, pp. 330-341, Feb. 2017.

[21] F. Declercq, H. Rogier and C. Hertleer, "Permittivity and Loss Tangent Characterization for Garment Antennas Based on a New Matrix-Pencil Two-Line Method," IEEE Transactions on Antennas and Propagation, vol. 56, no. 8, pp. 2548-2554, Aug. 2008.

[22] S. Liu, W. Wu and D. Fang, "Wideband Monopole-Like Radiation Pattern Circular Patch Antenna With High Gain and Low CrossPolarization," IEEE Trans. on Antennas and Propagation, vol. 64, no. 5, pp. 2042-2045, May 2016
[23] C. Delaveaud, P. Leveque and B. Jecko, ”New kind of microstrip antenna: the monopolar wire-patch antenna," Electron. Lett., vol. 30 , no. 1, pp. 1-2, 1994

[24] G. Conway, W. Scanlon, "Antennas for over-body-surface communication at $2.45 \mathrm{GHz}$," IEEE Trans. Antennas Propag., vol. 57, no. 4, pp. 844-855, Apr. 2009.

[25] J. Tak, S. Lee, and J. Choi, "All-textile higher order mode circular patch antenna for on-body to on-body communications," IET Microw., Antennas Propag., vol. 9, no. 6, pp. 576-584, Apr. 2015.

[26] R. Serra, D. Knittel, P. Di Croce and R. Peres, "Activity Recognition With Smart Polymer Floor Sensor: Application to Human Footstep Recognition," IEEE Sensors Journal, vol. 16, no. 14, pp. 5757-5775, Jul. 2016.

[27] C. Lu and L. Fu, "Robust Location-Aware Activity Recognition Using Wireless Sensor Network in an Attentive Home," IEEE Trans. on Automation Science and Engineering, vol. 6, no. 4, pp. 598-609, Oct. 2009.

[28] J. Liu, S. Zheng, Y. Li and Y. Long, "Broadband Monopolar Microstrip Patch Antenna With Shorting Vias and Coupled Ring," IEEE Antennas and Wireless Propagation Letters, vol. 13, pp. 39-42, Dec. 2014.

[29] "Flexfloors Stick Basic Black," Self-Adhesive PVC Flooring material, Part Nr. 585623, [Online] Available:http://www.flexxfloors.nl/. 